\title{
First bloodstream infection caused by Prevotella copri in a heart failure elderly patient with Prevotella-dominated gut microbiota: a case report
}

Patrizia Posteraro ${ }^{1 \dagger}$, Flavio De Maio ${ }^{2 \dagger}$, Giulia Menchinelli ${ }^{2}$, Ivana Palucci ${ }^{2}$, Federica Maria Errico ${ }^{1}$, Mariantonietta Carbone ${ }^{3}$, Maurizio Sanguinetti ${ }^{2,4^{*}} \mathbb{1}$, Antonio Gasbarrini $i^{5,6^{*}}$ and Brunella Posteraro 5,6

\begin{abstract}
Background: Bloodstream infection (BSI) is a constant threat for hospitalized patients, and elderly patients are particularly susceptible to BSI caused by anaerobic bacteria. Changes in the gut microbiota composition may lead to pathogen overgrowth and translocation into the bloodstream. Consequently, domination of specific taxa in the intestinal bacterial community seems to be associated with a higher risk of bacteremia in some patient populations.

Case presentation: Here, we report the case of a 90-year-old heart failure (HF) patient who was admitted to the hospital for an acute state of cardiac decompensation. Twenty days after admission, he was febrile to $38.2^{\circ} \mathrm{C}$ whereas his white blood count and C-reactive protein increased to 6190 cells $/ \mu \mathrm{L}$ and $31.2 \mathrm{mg} / \mathrm{L}$, respectively. Of the patient's blood culture (BC) bottle pairs collected under the suspicion of infection, the anaerobic bottle yielded an organism that was later identified as Prevotella copri. Concomitantly, the patient's fecal sample was obtained for the intestinal microbiota characterization by sequencing the V3N4N6 regions of the bacterial 16S rRNA gene. The analysis revealed highest relative abundances of Bacteroidales (34.1\%), Prevotellaceae (19.0\%), Prevotella (15.2\%), and P. copri (6.1\%) taxa, indicating that the patient's gut microbiota was dominated by Prevotella organisms. The patient was successfully treated with metronidazole, and was discharged to a long-term care facility at 35 days of admission.

Conclusions: We provide the first evidence for a clinically significant BSI caused by P. copri and its relationship to a Prevotella-rich gut microbiota in the HF patient setting. When strengthening the pathogenicity of P. copri, the present case suggests that the gut may be a source of BSI caused by the rare anaerobic organism. Future studies are necessary to assess the role of the gut microbiota profiling for precise identification and targeted treatment of patients at high risk of BSI.
\end{abstract}

Keywords: Bloodstream infection, Prevotella species, Microbiota, Heart failure, Precision medicine

\footnotetext{
*Correspondence: maurizio.sanguinetti@unicatt.it; antonio.

gasbarrini@unicatt.it

${ }^{\dagger}$ Patrizia Posteraro and Flavio De Maio contributed equally to this work

${ }^{2}$ Istituto di Microbiologia, Università Cattolica del Sacro Cuore, Rome, Italy

${ }^{5}$ Istituto di Patologia Medica e Semeiotica Medica, Università Cattolica

del Sacro Cuore, Rome, Italy

Full list of author information is available at the end of the article
}

(c) The Author(s) 2019. This article is distributed under the terms of the Creative Commons Attribution 4.0 International License (http://creativecommons.org/licenses/by/4.0/), which permits unrestricted use, distribution, and reproduction in any medium, provided you give appropriate credit to the original author(s) and the source, provide a link to the Creative Commons license, and indicate if changes were made. The Creative Commons Public Domain Dedication waiver (http://creativecommons.org/ publicdomain/zero/1.0/) applies to the data made available in this article, unless otherwise stated. 


\section{Background}

Bloodstream infection (BSI) is a constant menace for the hospitalized patients because of a number of risk factors related to underlying patient's diseases and/or conditions [1]. In particular, factors as cardiovascular disease, liver cirrhosis, diabetes mellitus, and cancer, either alone or concomitant with immunosuppressive therapies and/or presence of indwelling devices, concern patients in internal medicine hospital wards [2]. Consequent to unprecedented population aging, the today's patients are mostly elderly persons, and this makes their management particularly complex [3]. Since aged patients are at higher risk for infection because of more pronounced impairment of the anti-infective defenses, diagnosing BSI in such patients should require a high degree of suspicion $[2,3]$. Although bacteremia has long recognized as a common disorder in the elderly [4], presentations of bacteremia are often atypical because older patients tend to have fewer symptoms compared to younger patients, and this may unfavorably influence the BSI outcomes [3].

Elderly patients seem to have a predilection for BSIs caused by anaerobic bacteria [4], with anaerobes accounting for $2-5 \%$ of BSIs in most studies [3]. Bacteroides fragilis is the most common blood isolate recovered from adult patients with anaerobic bacteremia, whereas other species include Peptostreptococcus, Clostridium spp., and Fusobacterium spp; many of these infections are polymicrobial [5]. In a case series of Peptoniphilus spp. (i.e., Gram-positive anaerobic cocci formerly classified in the genus Peptostreptococcus) causing BSI in adults, three of 15 (20\%) of the case patients died, and all of them were elderly (age range 82-96 years) with significant underlying comorbidities [6]. While seven (47\%) of the 15 patients had polymicrobial BSIs, it is worthy to note that the recovery of Peptoniphilus alone, and not just as part of a polymicrobial infection, occurred in that study [6]. However, the clinical significance and the pathogenic potential of rare anaerobic bacteria in BSI remains undefined.

Besides having changed the clinicians' ideas about microbes in human health and disease [7], intense research into the gut microbiota has shed light on how the intestinal microbial community affects the susceptibility to infectious diseases [8]. In this context, recent studies have shown that the increased relative abundance (also termed intestinal "domination") of specific taxa in the gut bacterial communities (intestinal microbiota) enhanced the risk of bacteremia in some adult patient populations $[9,10]$. Thus, unspecified factors would contribute to the microbiota compositional changes and drive pathogen (also termed "pathobiont") overgrowth and translocation into the bloodstream [11]. Additionally, key association studies between specific changes in the intestinal microbiota composition and disease have shown that Blautia, Collinsella, unclassified Erysipelotrichaceae, and unclassified Ruminococcaceae significantly decreased in heart failure (HF) [12], whereas expansion of Prevotella copri correlated with predisposition to arthritis [13].

To our knowledge, the present report describes for the first time a symptomatic BSI caused by $P$. copri in a HF elderly patient, and relates this finding to the patient's gut microbiota dominated by Prevotella organisms.

\section{Case presentation}

A 90-year-old man was admitted to the Cardiology ward of the Ospedale San Carlo GVM of Rome, Italy, because of an acute state of cardiac decompensation. His presenting symptoms were weakness, shortness of breath, and peripheral edema and cyanosis. The patient had a long history of HF due to dilated cardiomyopathy, for which he had undergone to mitral valve repair. Three years before, he had a pacemaker implanted. The day of admission, on hospital day 1 (HD1), the echocardiography revealed that the mitral valve insufficiency was moderate whereas the tricuspid valve insufficiency was severe. The left ventricular ejection fraction (LVEF) was slightly reduced (52\%). According to current HF treatment guidelines [14], the patient was undergoing a drug treatment with angiotensin-converting enzyme inhibitor, beta-blocker, diuretic agent, aldosterone antagonist, and antiarrhythmic/heart rate modulating agent. He received levothyroxine and pantoprazole as further medications. Consistent with a previously diagnosed chronic renal failure, the patient's serum creatinine level was $2.48 \mathrm{mg} / \mathrm{dL}$ (reference range 0.70 to $1.20 \mathrm{mg} / \mathrm{dL})$. Serum marker measurement tests revealed a C-reactive protein (CRP) of $43.2 \mathrm{mg} / \mathrm{L}$ (reference range 0.0 to $<5.0 \mathrm{mg} / \mathrm{L}$ ) and an $\mathrm{N}$-terminal of the prohormone brain natriuretic peptide (NT-proBNP) of $33,826 \mathrm{pg} / \mathrm{mL}$ (reference range 0.0 to $<700.0 \mathrm{pg} / \mathrm{mL}$ ). A complete blood cell count test showed a white blood cell (WBC) count of 2830 cells $/ \mu \mathrm{L}$ (reference range 4000 to 10,000 cells $/ \mu \mathrm{L}$ ). The electrocardiogram did reveal no substantial acute alterations. Based on these findings, the patient's drug treatment remained unchanged. Fifteen days after admission, on HD16, the patient's serum CRP and NT-proBNP levels decreased to $6.5 \mathrm{mg} / \mathrm{L}$ and $4761 \mathrm{pg} / \mathrm{mL}$, respectively. Therefore, physicians planned the patient's transfer to a long-term care facility.

Twenty days after admission, on HD21, the patient was found to be febrile to $38.2{ }^{\circ} \mathrm{C}$, tachycardic, and tachypnic on physical exam. Laboratory tests revealed a WBC count of 6190 cells $/ \mu \mathrm{L}$, a CRP of $31.2 \mathrm{mg} / \mathrm{L}$, and an erythrocyte sedimentation rate of $49.0 \mathrm{~mm} / \mathrm{h}$ (reference range 0.0 to $12 \mathrm{~mm} / \mathrm{h}$ ). With the strong suspicion of infection, the patient's blood and other body sites were sampled for 
microbiological cultures. After 72-h incubation of blood culture (BC) bottles' (aerobic and anaerobic) pairs in the BACT/ALERT ${ }^{\circledR}$ 3D automated BC system (bioMérieux, Marcy l'Étoile, France), a single anaerobic bottle from the patient's $\mathrm{BC}$ set yielded a microorganism that appeared as a rod-shaped Gram-negative bacterium at the Gram stain microscopic observation. When subcultured on standard media, the bacterium grew only under anaerobic conditions. Pending the infecting-organism's identification results (see below), the patient started on empirical antimicrobial therapy with piperacillin-tazobactam and metronidazole.

Initially, the MALDI BioTyper ${ }^{\circledR}$ system (Bruker Daltonics, Bremen, Germany) based identification provided a Prevotella stercorea DSM 18206T organism as the third best match hit against the MALDI reference database, with an identification score value of 1.400 (not reliable identification score, $\leq 1.699$; reliable identification score, $>1.699)$. The first and second match hits were Paracoccus versutus B352 UFL (score value, 1.505) and Staphylococcus capitis DSM 20325 (score value, 1.438). Then, the Prevotella organism was reliably identified as P. copri through comparing the partial $16 \mathrm{~S}$ ribosomal RNA (rRNA) gene sequence (ABI Prism 3130 sequencer; Applied Biosystems, Foster City, CA) against the NCBI database using BLAST, that yielded $>99 \%$ identity with the $P$. copri sequence (GenBank accession, MG592701.1). In the meantime, we obtained a patient's fecal sample for the 16S rRNA gene-targeted intestinal microbiota profiling (see Additional file 1: Methods). As shown in Table 1, sequencing of the V3, V4, and V6 regions of 16S rRNA gene showed highest relative abundances for the Bacteroidales order (34.1\%), Prevotellaceae family (19.0\%), Prevotella genus (15.2\%), and P. copri species (6.1\%). Interestingly, together with other Prevotella species (DJF RP53, BI-42, and DJF B112), P. copri accounted for $10.5 \%$ of all the intestinal microbiota composing (classified and unclassified) species. All these taxa belong to the Bacteroidetes phylum, which was the second most abundant (34.6\%) after the Firmicutes phylum (37.0\%) in the patient's intestinal microbiota.

On HD26, the patient switched to metronidazole alone while subsequently collected BCs became negative. On HD35, he was hemodynamically stable and was finally discharged to a long-term care facility.

\section{Discussion and conclusions}

The present case's patient is the archetype of internal medicine patients in whom multiple comorbidities, polypharmacy, and length of hospitalization may concur to the acquisition of BSI [2]. With regard to HF (the main patient's comorbidity), it should be recalled that the disease is not simply a cardiac disease but rather a complex multi-organ clinical syndrome [15]. In view of the new disease concepts [16], intestinal dysbiosis-a state of microbial community imbalance-in HF [12] could not only represent a key pathogenic factor but also mediate the leakage of the immune barrier, leading to chronic inflammatory disorders [17]. Until now, no studies have shown the potential of $P$. copri to break down the intestinal barrier [18], which may indeed trigger systemic inflammation through the microbial or endotoxin translocation to the systemic circulation in decompensated HF patients [19].

Table 1 Most relatively abundant bacteria on order, family, genus, and species level identified in the patient's fecal sample

\begin{tabular}{|c|c|c|c|}
\hline Taxa & \% abundance & Taxa & $\%$ abundance \\
\hline Order & & Genus & \\
\hline Bacteroidales & 34.114 & Prevotella & 15.253 \\
\hline Clostridiales & 27.590 & Faecalibacterium & 8.215 \\
\hline \multirow[t]{3}{*}{ Selenomonadales } & 7.493 & Dialister & 6.372 \\
\hline & & Parabacteroides & 3.849 \\
\hline & & Bacteroides & 3.750 \\
\hline Family & & Species & \\
\hline Prevotellaceae & 19.024 & Prevotella copri & 6.133 \\
\hline Ruminococcaceae & 16.519 & Dialister succinatiphilus & 5.869 \\
\hline Lachnospiraceae & 9.157 & Faecalibacterium prausnitzii & 3.180 \\
\hline Porphyromonadaceae & 6.999 & Parabacteroides distasonis & 2.488 \\
\hline Veillonellaceae & 6.758 & Prevotella sp. (DJF RP53) & 2.050 \\
\hline Bacteroidaceae & 3.750 & & \\
\hline
\end{tabular}

The listed taxa belong to the Firmicutes and Bacteroidetes phyla, whose relative abundances were $37.054 \%$ and $34.664 \%$, respectively. Unlisted species include two other Prevotella sp. (BI-42 and DJF B112), whose relative abundances were $1.504 \%$ and $0.836 \%$, respectively 
Here, we provided the first evidence that a Prevotellarich intestinal microbiota relates to the onset of a clinically significant BSI caused by P. copri in the HF patient setting, thereby supporting the growing emphasis on $P$. copri-and Prevotella species in general-as a pathobiont but not as a beneficial bacterium [20-22]. The relative abundance observed for Prevotellaceae in our patient is lower than the relative abundance percentages reported as threshold values for the increased risk of Enterococcaceae, Streptococcaceae, and Proteobacteria bacteremia in hematopoietic stem cell transplantation or long-term acute care hospital patients $[9,10]$. Unlike in our case, patients in the two studies have common risk factors for bacterial translocation in the gut $[9,10]$, and one study apply a threshold of $22 \%$ relative abundance to predict bacteremia caused by Klebsiella pneumoniae carbapenemase-producing Klebsiella pneumoniae (КРC-Kp) [10]. Additionally, association between exposure to certain antibiotics (i.e., those more strongly associated with clinically important microbiota disruption) and subsequent sepsis within 90 days after a hospital stay has recently been documented [23]. In our case, the patient did not receive antibiotics during the hospital stay before the BSI onset nor have any of predisposing factors to anaerobic bacteremia, which include malignant neoplasms, hematologic disorders, organ transplantation, diabetes mellitus, and an undrained abscess among the others [5]. Therefore, it is plausible that the occupation of at least $15 \%$ of our patient's fecal microbiota by Prevotella as a single predominating bacterial taxon would have allowed P. copri to cross the intestinal barrier and then became detectable in the patient's blood. Yet, it is unclear if the long-term administration of proton-pump inhibitor could have altered the gut microbiota composition in our patient. In accordance with us, Prevotella had a relative abundance between 10 and $40 \%$ in $16 \%$ of microbiota samples analyzed in one study, but $75 \%$ of samples had a relative abundance less than 5\% [24]. Because differences in the samples' Prevotella (and Bacteroides) abundances did not represent consistent microbial communities within the "enterotypes" described originally [20], Gorvitovskaia et al. proposed to use "biomarker" for representing the dominant taxon of a given microbial community [20]. Thus, we can interpret Prevotella not only as a "biomarker" of diet or lifestyle [20] but also of disease state.

This case shows that physicians should suspect anaerobes as the cause of bacteremia not only based on typical clinical predictors [5] and, especially in patients with complex underlying disease, should require BCs supporting the growth of anaerobic bacteria always and when not only an anaerobic infection is present or suspected clinically [5]. Like other Gram-negative bacilli, Prevotella species are non-spore-forming, non-motile, singular cells that thrive under anaerobic growth conditions. Therefore, their isolation from the patient's BCs-the today's laboratory diagnostic standard for BSI-is somewhat difficult because of prolonged organism's growth times that often exceed the conventional 5-day incubation $\mathrm{BC}$ period [25]. Our patient presented with fever $\left(>38{ }^{\circ} \mathrm{C}\right)$ which represents a typical symptom of bacteremia [3, 5]. By reviewing studies that evaluated aged (65 years and older) patients hospitalized with BSIs, Yahav et al. [3] reported that the fever was present in at least $75 \%$, whereas leukocytosis in $39-73 \%$ and leukopenia in $~ 10 \%$, of the patients studied. In one of these studies, elevation of CRP was as common in older patients as in younger patients [26]. In our patient, increased levels of either WBC (twofold) or CRP (fivefold) occurred immediately prior to the BSI onset. This strengthens the pathogenicity of the P. copri isolate and, importantly, counterbalances the fact we were unable to recover the isolate from more than one blood sample of our patient, which is often the case in many instances [5]. Consistent with the fact that anaerobic bacteria including Prevotella species are slow and/or fastidious growing on standard subculture media, we could not obtain reliable identification of the patient's isolate with the MALDI BioTyper ${ }^{\circledR}$ system, which is a well-suited method in routine laboratories [27]. As anaerobic Gram-negative isolates can be resistant to penicillin, a combination of a beta-lactamase inhibitor and a penicillin may be appropriate in cases of infection caused by $B$. fragilis group, Fusobacterium species, or pigmented Prevotella species [5]. Thus, based on the positivity of the patient's anaerobic $\mathrm{BC}$ results physicians selected a combination of piperacillin-tazobactam and metronidazole to treat empirically the patient. Following the availability of the isolate's final identification by the PCR sequencing method, physicians shifted the patient's antimicrobial treatment to only metronidazole, and this treatment was successful.

In conclusion, we documented the first clinically significant recovery of $P$. copri from the blood of a HF patient. At the same time, we detected $P$. copri as the relatively most abundant organism in the gut of the patient, which strongly indicates a relationship between $P$. copri as a cause of primary BSI and an intestinal microbiota dominated by the same organism. Although we did not show that intestinal dominance by the BSI pathogen preceded the onset of infection [28], the assumption of a higher relative abundance of $P$. copri in the patient's gut prior to infection is reasonable. Finally, our case underlines the importance of profiling the gut microbiota in patients particularly at risk of BSI to allow a precise identification and targeted treatment of these patients. In this context, the metronidazole prophylaxis to cardiac patients undergoing any interventions would be extended to routine 
administration on hospitalization if their gut microbiota was proven to be altered.

\section{Supplementary information}

Supplementary information accompanies this paper at https://doi. org/10.1186/s13099-019-0325-6.

Additional file 1. Methods for the 16 rRNA gene based intestinal microbiota characterization.

\section{Abbreviations}

BSI: bloodstream infection; HF: heart failure; HD: hospital day; LVEF: left ventricular ejection fraction; CRP: C-reactive protein; NT-proBNP: N-terminal of the prohormone brain natriuretic peptide; WBC: white blood cell; BC: blood culture; MALDI: matrix-assisted laser desorption ionization; rRNA: ribosomal RNA.

\section{Acknowledgements}

We would like thank Giulia De Angelis and Lara Boatti for critical reading of the manuscript.

\section{Authors' contributions}

PP and FDM conceived the study and analyzed all data. PP and BP wrote the manuscript. FDM and GM prepared and ran the sequencing. IP performed conventional and molecular identification analyses. FME performed routine microbiology analyses. MC analyzed and interpreted the patient's data regarding the underlying disease. MS and AG supervised the data analysis and helped write the paper. All authors read and approved the final manuscript.

\section{Funding}

This study was not funded.

\section{Availability of data and materials}

The partial 16S rRNA gene sequence of the patient's isolate is available in the GenBank database under the accession number MK680126.

The datasets analyzed during this study are available in the NCBI Sequence Read Archive repository [PRJNA529053] (https://www.ncbi.nlm.gov/bioproject 1529053).

\section{Ethics approval and consent to participate}

Not applicable.

\section{Consent for publication}

Written informed consent for publication of this case report was obtained from the patient.

\section{Competing interests}

The authors declare that they have no competing interests.

\section{Author details}

${ }^{1}$ Laboratorio di Analisi Chimico-Cliniche e Microbiologiche, Ospedale San Carlo GVM, Rome, Italy. ${ }^{2}$ Istituto di Microbiologia, Università Cattolica del Sacro Cuore, Rome, Italy. ${ }^{3}$ Unità di Cardiologia, Ospedale San Carlo GVM, Rome, Italy. ${ }^{4}$ Dipartimento di Scienze di Laboratorio e Infettivologiche, Fondazione Policlinico Universitario A. Gemelli IRCCS, Rome, Italy. ${ }^{5}$ Istituto di Patologia Medica e Semeiotica Medica, Università Cattolica del Sacro Cuore, Rome, Italy. ${ }^{6}$ Dipartimento di Scienze Gastroenterologiche, Endocrino-Metaboliche e Nefro-Urologiche, Fondazione Policlinico Universitario A. Gemelli IRCCS, Rome, Italy.

Received: 19 June 2019 Accepted: 12 September 2019

Published online: 18 September 2019

\section{References}

1. Boev C, Kiss E. Hospital-acquired infections: current trends and prevention. Crit Care Nurs Clin N Am. 2017;29:51-65.
2. Del Bono V, Giacobbe DR. Bloodstream infections in internal medicine. Virulence. 2016;7:353-65.

3. Yahav D, Eliakim-Raz N, Leibovici L, Paul M. Bloodstream infections in older patients. Virulence. 2016;7:341-52.

4. Leibovici L. Bacteraemia in the very old. Features and treatment. Drugs Aging. 1995;6:456-64.

5. Brook I. The role of anaerobic bacteria in bacteremia. Anaerobe 2010;16:183-9.

6. Brown K, Church D, Lynch T, Gregson D. Bloodstream infections due to Peptoniphilus spp.: report of 15 cases. Clin Microbiol Infect. 2014:20:0857-60

7. Young VB. The role of the microbiome in human health and disease: an introduction for clinicians. BMJ. 2017;356:j831.

8. Libertucci J, Young VB. The role of the microbiota in infectious diseases. Nat Microbiol. 2019;4:35-45.

9. Taur Y, Xavier JB, Lipuma L, Ubeda C, Goldberg J, Gobourne A, Lee YJ, Dubin KA, Socci ND, Viale A, Perales MA, Jeng RR, van den Brink MR, Pamer EG. Intestinal domination and the risk of bacteremia in patients undergoing allogeneic hematopoietic stem cell transplantation. Clin Infect Dis. 2012;55:905-14.

10. Shimasaki T, Seekatz A, Bassis C, Rhee Y, Yelin RD, Fogg L, Dangana T, Cisneros EC, Weinstein RA, Okamoto K, Lolans K, Schoeny M, Lin MY, Moore NM, Young VB, Hayden MK, Centers for Disease Control and Prevention Epicenters Program. Increased relative abundance of Klebsiella pneumoniae carbapenemase-producing Klebsiella pneumoniae within the gut microbiota is associated with risk of bloodstream infection in long-term acute care hospital patients. Clin Infect Dis. 2018. https://doi.org/10.1093/ cid/ciy796 (Epub ahead of print).

11. Kamada N, Chen GY, Inohara N, Núñez G. Control of pathogens and pathobionts by the gut microbiota. Nat Immunol. 2013;14:685-90.

12. Luedde M, Winkler T, Heinsen FA, Rühlemann MC, Spehlmann ME, Bajrovic A, Lieb W, Franke A, Ott SJ, Frey N. Heart failure is associated with depletion of core intestinal microbiota. ESC Heart Fail. 2017;4:282-90.

13. Scher JU, Sczesnak A, Longman RS, Segata N, Ubeda C, Bielski C, Rostron T, Cerundolo V, Pamer EG, Abramson SB, Huttenhower C, Littman DR. Expansion of intestinal Prevotella copri correlates with enhanced susceptibility to arthritis. Elife. 2013;2:e01202.

14. Ponikowski P, Voors AA, Anker SD, Bueno H, Cleland JG, Coats AJ, Falk V, González-Juanatey JR, Harjola VP, Jankowska EA, Jessup M, Linde C, Nihoyannopoulos P, Parissis JT, Pieske B, Riley JP, Rosano GM, Ruilope LM, Ruschitzka F, Rutten FH, van der Meer P, Authors/Task Force Members; Document Reviewers. 2016 ESC Guidelines for the diagnosis and treatment of acute and chronic heart failure: The Task Force for the diagnosis and treatment of acute and chronic heart failure of the European Society of Cardiology (ESC). Developed with the special contribution of the Heart Failure Association (HFA) of the ESC. Eur J Heart Fail. 2016;18:891-975.

15. Warriner $D$, Sheridan $P$, Lawford P. Heart failure: not a single organ disease but a multisystem syndrome. Br J Hosp Med. 2015;76:330-6.

16. Nagatomo $\mathrm{Y}$, Tang $\mathrm{WH}$. Intersections between microbiome and heart failure: revisiting the gut hypothesis. J Card Fail. 2015;21:973-80.

17. Kamada N, Seo SU, Chen GY, Núñez G. Role of the gut microbiota in immunity and inflammatory disease. Nat Rev Immunol. 2013;13:321-35.

18. Maeda Y, Takeda K. Role of gut microbiota in rheumatoid arthritis. J Clin Med. 2017;6:E60.

19. Sandek A, Bjarnason I, Volk HD, Crane R, Meddings JB, Niebauer J, Kalra PR, Buhner S, Herrmann R, Springer J, Doehner W, von Haehling S, Anker SD, Rauchhaus M. Studies on bacterial endotoxin and intestinal absorption function in patients with chronic heart failure. Int J Cardiol. 2012;157:80-5.

20. Arumugam M, Raes J, Pelletier E, Le Paslier D, Yamada T, Mende DR, Fernandes GR, Tap J, Bruls T, Batto JM, Bertalan M, Borruel N, Casellas F, Fernandez L, Gautier L, Hansen T, Hattori M, Hayashi T, Kleerebezem M, Kurokawa K, Leclerc M, Levenez F, Manichanh C, Nielsen HB, Nielsen T, Pons N, Poulain J, Qin J, Sicheritz-Ponten T, Tims S, Torrents D, Ugarte E, Zoetendal EG, Wang J, Guarner F, Pedersen O, de Vos WM, Brunak S, Doré J, MetaHIT Consortium, Antolín M, Artiguenave F, Blottiere HM, Almeida M, Brechot C, Cara C, Chervaux C, Cultrone A, Delorme C, Denariaz G, Dervyn R, Foerstner KU, Friss C, van de Guchte M, Guedon E, Haimet F, Huber W, van Hylckama-Vlieg J, Jamet A, Juste C, Kaci G, Knol J, Lakhdari O, Layec S, Le Roux K, Maguin E, Mérieux A, Melo Minardi R, M'rini C, Muller J, Oozeer R, Parkhill J, Renault P, Rescigno M, Sanchez N, Sunagawa 
S, Torrejon A, Turner K, Vandemeulebrouck G, Varela E, Winogradsky Y, Zeller G, Weissenbach J, Ehrlich SD, Bork P. Enterotypes of the human gut microbiome. Nature. 2011;473:174-80.

21. Kim D, Kim WU. Can Prevotella copri be a causative pathobiont in rheumatoid arthritis? Arthritis Rheumatol. 2016;68:2565-7.

22. Armstrong AJS, Shaffer M, Nusbacher NM, Griesmer C, Fiorillo S, Schneider JM, Preston Neff C, Li SX, Fontenot AP, Campbell T, Palmer BE, Lozupone CA. An exploration of Prevotella-rich microbiomes in HIV and men who have sex with men. Microbiome. 2018:6:198.

23. Baggs J, Jernigan JA, Halpin AL, Epstein L, Hatfield KM, McDonald LC. Risk of subsequent sepsis within 90 days after a hospital stay by type of antibiotic exposure. Clin Infect Dis. 2018;66:1004-12.

24. Gorvitovskaia A, Holmes SP, Huse SM. Interpreting Prevotella and Bacteroides as biomarkers of diet and lifestyle. Microbiome. 2016;4:15.

25. Fiori B, D'Inzeo T, Giaquinto A, Menchinelli G, Liotti FM, de Maio F, De Angelis G, Quaranta G, Nagel D, Tumbarello M, Posteraro B, Sanguinetti M, Spanu T. Optimized use of the MALDI BioTyper system and the FilmArray
BCID panel for direct identification of microbial pathogens from positive blood cultures. J Clin Microbiol. 2016;54:576-84.

26. Wester AL, Dunlop O, Melby KK, Dahle UR, Wyller TB. Age-related differences in symptoms, diagnosis and prognosis of bacteremia. BMC Infect Dis. 2013;13:346.

27. Nagy E, Boyanova L, Justesen US, ESCMID Study Group of Anaerobic Infections. How to isolate, identify and determine antimicrobial susceptibility of anaerobic bacteria in routine laboratories. Clin Microbiol Infect. 2018;24:1139-48.

28. Tamburini FB, Andermann TM, Tkachenko E, Senchyna F, Banaei N, Bhatt AS. Precision identification of diverse bloodstream pathogens in the gut microbiome. Nat Med. 2018;24:1809-14.

\section{Publisher's Note}

Springer Nature remains neutral with regard to jurisdictional claims in published maps and institutional affiliations.
Ready to submit your research? Choose BMC and benefit from:

- fast, convenient online submission

- thorough peer review by experienced researchers in your field

- rapid publication on acceptance

- support for research data, including large and complex data types

- gold Open Access which fosters wider collaboration and increased citations

- maximum visibility for your research: over $100 \mathrm{M}$ website views per year

At BMC, research is always in progress.

Learn more biomedcentral.com/submissions 